\title{
Image generation in a letter-classification task
}

\author{
THOMAS R. HERZOG \\ Grand Valley State Colleges, Allandale, Michigan 49401
}

\begin{abstract}
Subjects classified briefly presented uppercase letters as belonging to the first or second half of the alphabet. A prior alternative preceded each target letter by 200 or 1,400 msec. It was either an ampersand (control), a lowercase letter (opposite case), or an uppercase letter (same case). In the latter two conditions, the target was equally likely to be identical in name to the prior alternative or from the opposite half of the alphabet. For the 200 -msec onset asynchrony, classification accuracy was greater in the same-case condition than in the other two conditions, which did not differ. For the 1,400-msec onset asynchrony, accuracy in both the same- and opposite-case conditions was greater than accuracy in the control condition. Same- and opposite-case prior alternatives always biased subjects toward classifying the target in the same half of the alphabet as the alternative. The accuracy results suggest that internally generated images can be used to aid target classification in the opposite-case condition.
\end{abstract}

Posner (1969, 1973a; Posner, Boies, Eichelman, \& Taylor, 1969) has provided evidence for what he calls "image generation" in a reaction-time task. In these studies, the subject saw a single letter followed, after a variable interval, by a second letter. The subject pressed one switch as rapidly as possible if the two letters had the same name, another switch if they had different names. Of special interest are two conditions: in one the two letters were always uppercase, and in the other, the second letter was always opposite in case to the first. For short stimulus onset asynchronies, same responses had shorter reaction times in the same-case condition. However, for onset asynchronies of about 1-2 sec, same responses in the opposite-case condition had become just as fast as in the same-case condition. Posner concluded that in the opposite-case condition subjects generated an internal image of the opposite case of the first letter to use in matching against the target and that a few seconds were required to complete the generation process.

Can such a process be demonstrated when the second letter is presented very briefly and an accuracy measure is used instead of reaction time? The experiments reported here investigated this question. It is of interest because accuracy data obtained under marginal viewing conditions and reaction times obtained under relatively favorable viewing conditions do not always yield similar results for a given independent variable (e.g., Skanes \& Donderi, 1973).

A satisfactory demonstration of the generation process must explore its temporal boundaries. It must show not only that generation has time to occur with long onset asynchronies, but also that it does

This research was supported by a grant from the Grand Valley State Colleges Research Development Fund to the author. Requests for reprints should be sent to Thomas R. Herzog, Department of Psychology, Grand Valley State Colleges, Allendale, Michigan 49401. not have time to occur when onset asynchronies are too brief. Although several studies using accuracy measures of performance in marginal viewing conditions have obtained results compatible with an imagegeneration process (Egeth \& Smith, 1967; Gummerman, 1971; Long, Reid, \& Henneman, 1960; Taylor \& Reilly, 1970), few have examined both short and long onset asynchronies. Herzog (1976) looked at an "immediate" and a "delayed" condition, but his onset asynchronies were not precisely controlled. Pachella (1975) used two onset asynchronies, but both were quite long (6 and $15 \mathrm{sec}$ ) relative to the asynchronies in the Posner studies. Despite these methodological reservations, both studies obtained results that could be interpreted in terms of image generation.

In the present experiments, the basic task was the classification of briefly presented single uppercase letters as belonging to the first or the second half of the alphabet. Each trial consisted of a foreperiod followed immediately by the target letter. The foreperiod contained an ampersand (control), a lowercase letter, or an uppercase letter. In the latter two conditions, the subject could derive the correct answer by successfully matching the target letter against the uppercase version of the prior-alternative letter. When the prior-alternative letter was lowercase, such matching would require the subject to generate the uppercase version. The onset asynchrony of the prioralternative symbol and the target letter was varied across experiments.

The control condition provided a baseline against which performance with same- and opposite-case prior alternatives could be compared at short and long onset asynchronies. If image generation does not have time to occur with a short onset asynchrony, then there should be no difference in classification accuracy between the opposite-case and control 
conditions. However, with a long onset asynchrony, accuracy in the opposite-case condition should be significantly superior to that in the control condition. Since the same-case condition does not require image generation for successful matching, accuracy in the same-case condition should be superior to that in the control condition for both short and long onset asynchronies. Experiment 1 tested the predictions for a short onset asynchrony $(200 \mathrm{msec})$. Experiment 2 tested the predictions for a long onset asynchrony $(1,400 \mathrm{msec})$.

\section{METHOD}

\section{Subjects}

The subjects were female undergraduate volunteers with normal or corrected-to-normal vision. Participation satisfied an introductory psychology course requirement. A different sample of 14 subjects was run in each experiment. Each subject served in four 1-h sessions, except for one subject in each experiment who failed to appear for her last session. The scores for those subjects were based on their responses in the earlier sessions.

\section{Apparatus}

The target stimuli were eight uppercase letters (A, C, K, L, N, $S, U, Z$ ), four from each half of the alphabet. The prior-alternative stimuli were the same eight uppercase letters, their lowercase versions, and an ampersand. All stimuli consisted of "Letraset" black dry-transfer letters (Futura Bold, 20-point) mounted on plain white mat-surface index cards. At $91-\mathrm{cm}$ viewing distance, the uppercase letters and the ampersand subtended $21 \mathrm{~min}$ of viewing angle vertically, and the lowercase letters subtended from 14 to $21 \mathrm{~min}$ of viewing angle. Target letters were presented in one field of a three-field tachistoscope (Iconix, Inc.), prior-alternative symbols in a second field, and a fixation dot in the third field. The fixation dot was located slightly to the left of the center of the viewing area and was present whenever another stimulus was not being shown. The stimulus cards for the prior alternatives each contained a dot that appeared in the same location, and the prior-alternative symbols appeared $1.90 \mathrm{~cm}$ to the right of the dot. The target letters appeared at the location of the dot. Subjects were instructed to maintain fixation on the dot throughout each trial and not to look directly at the prior-alternative symbol. This was done to prevent the afterimage of the prior alternative from interfering with the perception of the target letter. The three fields were adjusted to appear equally bright to the subject during a trial. Luminances averaged $18.93 \mathrm{~cd} / \mathrm{m}^{2}$ for the target field, $18.03 \mathrm{~cd} / \mathrm{m}^{2}$ for the prior-alternative field, and $16.97 \mathrm{~cd} / \mathrm{m}^{2}$ for the background field. Viewing was binocular.

\section{Procedure}

In each session, a modified staircase procedure was used to determine an exposure duration at which the subject could correctly classify a target letter in the control condition about $62.5 \%$ of the time. That exposure duration was used for all target letters during the next three blocks of trials which constituted one replication of the experiment. Then, if necessary, an adjustment in exposure duration was made, followed by another replication of the experiment consisting of three blocks of trials. Exposure durations ranged from 1.1 to $6.0 \mathrm{msec}$.

Each trial consisted of a foreperiod followed immediately and automatically by a target letter. The duration of the foreperiod was $200 \mathrm{msec}$ in Experiment $1,1,400 \mathrm{msec}$ in Experiment 2. The duration of the prior-alternative symbol was always $200 \mathrm{msec}$, coinciding with the foreperiod in Experiment 1 and occupying the first $200 \mathrm{msec}$ of the foreperiod in Experiment 2. To equate for temporal uncertainty with respect to the onset of the target letter, a pure tone of 100 -msec duration was presented $1,100 \mathrm{msec}$ after the onset of the foreperiod in Experiment 2. The subject was instructed to regard the tone as a signal that the target letter would occur a fraction of a second later. In both experiments, the subject initiated each trial by pressing a button in response to the experimenter's verbal ready signal. After each trial, the subject responded verbally and in writing, indicating which half of the alphabet ("one" or "two") the target letter came from. The intertrial interval was approximately $3-4 \mathrm{sec}$. The subject was given trial-by-trial feedback in the first session (a practice session, data not analyzed) but no feedback in the last three sessions.

Each of the last three sessions contained 108 experimental trials, grouped into six blocks of 18 trials each. Each block was devoted to a different one of three experimental conditions.

\section{Control}

The prior-alternative symbol was always an ampersand.

\section{Opposite Case}

The prior alternative was the lowercase version of one of the eight letters.

\section{Same Case}

The prior alternative was the uppercase version of one of the eight letters.

In the same- and opposite-case conditions, the target letter was equally likely to be the uppercase version of the prior-alternative letter (same trials) or the uppercase version of one of the four letters from the opposite half of the alphabet (different trials). The subject was informed in advance of this arrangement and was specifically told that she could determine the correct response in these conditions simply by deciding whether or not the target letter was the same as the uppercase version of the prior-alternative letter. However, as indicated, the subject always responded by saying "one" or "two." The subject was told that each of the eight letters was equally likely as the target on each trial in the control condition.

Trial-order lists for three complete sessions were prepared in advance. Each subject used a different list in each of her last three sessions, with order of presentation of the lists balanced across subjects. Each list consisted of six blocks of 16 trials, two blocks for each experimental condition. The 16 trials consisted of 8 same trials and 8 different trials. Same and different trials were designated even for the control condition (although the prioralternative letter was never presented) so that an implicit samedifferent response bias could be calculated for all conditions (see Scoring section). Within each trial block, assignment of stimuli to trials was random, except that no two consecutive trials with the same letter and no more than four consecutive same or different trials or trials with letters from the same half of the alphabet were allowed. Also, within each list, the eight letters appeared equally often as target letters and as prior-alternative letters in each experimental condition. The first three trial blocks within a list were each assigned to a different experimental condition, with order of presentation of the three conditions balanced across lists. For the last three trial blocks of each list, the three experimental conditions appeared in reverse order from the first three trial blocks of that list.

\section{Scoring}

For each subject, a measure of classification accuracy $\left(A^{\prime}\right)$ and a measure of response bias $\left(B_{H}^{\prime}\right)$ were derived. Correct responses on same trials were scored as hits, and incorrect responses on different trials were scored as false alarms. The hit and false-alarm rates for each subject in each condition were converted to the nonparametric sensitivity index $A^{\prime}$ and the nonparametric responsebias index $B^{\prime}{ }_{H}$ using Grier's (1971) formulas. A negative $B^{\prime}{ }_{H}$ indicates a bias toward classifying the target in the same half of the alphabet as the prior alternative, and a positive $\mathrm{B}^{\prime}{ }_{\mathrm{H}}$ indicates a bias toward classifying the target in the opposite half of the alphabet. In these experiments, classifying the target in the same half of the alphabet as the prior alternative was equivalent to saying that 
the two were the same letter (see Procedure section). Likewise, classifying the target in the opposite half of the alphabet from the prior alternative was equivalent to saying that the two were different letters. Since the subjects never said "same" or "different," but those words were implicit in what they did say, the $B^{\prime}{ }_{H}$ scores may be considered measures of an implicit bias toward sameness or difference.

\section{RESULTS}

Table 1 presents the mean sensitivity $\left(A^{\prime}\right)$ and response-bias $\left(\mathbf{B}^{\prime}{ }_{H}\right)$ scores for both experiments. Since specific comparisons among conditions were of interest and could be stated in advance, data analysis was by means of planned comparisons. Individual error terms were computed for each comparison, as recommended by Keppel (1973) for within-subjects designs. For each set of means, two orthogonal comparisons were possible. For the $\mathrm{A}^{\prime}$ means, one comparison was between the control and the oppositecase conditions. Since image generation was thought to be possible only with the 1,400 -msec onset asynchrony, this comparison was expected to be significant in Experiment 2 but not in Experiment 1. The other comparison was between the combined control and opposite-case conditions and the same-case condition. Since the same-case condition was predicted to yield superior performance at all onset asynchronies, this comparison was expected to be significant in both experiments. For the $\mathrm{B}^{\prime}{ }_{\mathrm{H}}$ means, one comparison was between the control condition and the combined opposite- and same-case conditions. From earlier work (Herzog, 1976), it was expected that either type of prior alternative would induce a bias toward classifying the target in the same half of the alphabet as the alternative, but no such bias should occur in the control condition. Hence, this comparison was expected to be significant. The other comparison was between the opposite-case condition and the samecase condition. The object was to see if either type of prior alternative induced a stronger implicit bias toward sameness, and no specific prediction was made.

\section{Experiment 1}

For the $A^{\prime}$ means of Experiment 1 (Table 1, Row 1), the comparison between the control and

Table 1

Mean Sensitivity $\left(A^{\prime}\right)$ and Response-Bias $\left(B^{\prime}{ }_{H}\right)$ Scores as a Function of Experimental Condition for Experiments 1 and 2

\begin{tabular}{|c|c|c|c|c|}
\hline \multirow[b]{2}{*}{$\begin{array}{c}\text { Onset } \\
\text { Asynchrony } \\
\end{array}$} & \multirow[b]{2}{*}{$\begin{array}{l}\text { Performance } \\
\text { Measure }\end{array}$} & \multicolumn{3}{|c|}{ Experimental Condition } \\
\hline & & Control & $\begin{array}{l}\text { Opposite } \\
\text { Case }\end{array}$ & $\begin{array}{l}\text { Same } \\
\text { Case }\end{array}$ \\
\hline \multicolumn{5}{|c|}{ Experiment 1} \\
\hline $200 \mathrm{msec}$ & $\begin{array}{l}\mathrm{A}^{\prime} \\
\mathrm{B}^{\prime}{ }_{\mathrm{H}}\end{array}$ & $\begin{array}{l}.62 \\
.01\end{array}$ & $\begin{array}{r}.65 \\
-.12\end{array}$ & $\begin{array}{r}.72 \\
-.29\end{array}$ \\
\hline \multicolumn{5}{|c|}{ Experiment 2} \\
\hline $1400 \mathrm{msec}$ & $\begin{array}{l}\mathbf{A}^{\prime} \\
\mathbf{B}^{\prime}{ }_{H}\end{array}$ & $\begin{array}{l}.64 \\
.00\end{array}$ & $\begin{array}{r}.70 \\
-.21\end{array}$ & $\begin{array}{r}.74 \\
-.24\end{array}$ \\
\hline
\end{tabular}

opposite-case conditions was not significant $[\mathrm{F}(1,13)$ $=.58, \mathrm{p}>.05, \mathrm{MSe}=.0075]$. Only 8 of the 14 subjects had larger $A^{\prime}$ scores in the opposite-case condition than in the control condition. The comparison between the combined control and opposite-case conditions and the same-case condition was significant $[F(1,13)=13.77, p<.005, \mathrm{MSe}=.0049]$. Since the control and opposite-case means did not differ, it is reasonable to conclude that the same-case mean was significantly larger than the control mean. In fact, the $A^{\prime}$ scores for 12 of the 14 subjects were larger in the same-case condition than in the control condition. Thus, same-case prior alternatives enhanced classification accuracy; opposite-case prior alternatives did not.

For the $\mathrm{B}_{\mathrm{H}}$ means (Table 1, Row 2), the comparison between the control condition and the combined opposite- and same-case conditions was significant $[\mathrm{F}(1,13)=14.72, \mathrm{p}<.005$, MSe $=.0302]$. The largest $B^{\prime}{ }_{H}$ score occurred in the control condition for 11 of the 14 subjects. The comparison between the opposite- and same-case conditions was not significant $[\mathrm{F}(1,13)=3.87, \mathrm{p}>.05, \mathrm{MSe}=.0475]$, although it was close $(p<.10)$. Of the 14 subjects, 9 had larger $\mathbf{B}^{\prime}{ }_{H}$ scores (indicating less bias) in the opposite-case condition. The hypothesis of no bias was evaluated separately in each condition by $t$ tests. The results indicated a significant implicit bias toward sameness in the same-case condition $(p<.05)$, a tendency toward the same kind of bias in the oppositecase condition $(p<.10)$, and no bias in the control condition. Thus, both same- and opposite-case prior alternatives tended to bias the subject toward indicating that the target letter was the same as the prior alternative. It is unclear whether either type of alternative induced a stronger bias.

The data in both experiments were also scored in terms of the actual response categories of the task. That is, correct responses to targets in the first half of the alphabet ("one') were scored as hits, and incorrect responses to targets in the second half of the alphabet ("one") were scored as false alarms. $A^{\prime}$ scores derived from these hit and false-alarm rates were necessarily quite similar to the same-different $A^{\prime}$ scores because the percentage of correct classifications is the same using either scoring system. The $\mathbf{B}^{\prime}{ }_{\mathrm{H}}$ scores were quite different, since the scoring system described here assesses the bias toward saying "one" or "two." The results indicated no such bias in any of the conditions of either experiment.

\section{Experiment 2}

For the $\mathbf{A}^{\prime}$ means of Experiment 2 (Table 1, Row 3), the comparison between the control and opposite-case conditions was significant $[F(1,13)=$ $10.69, \mathrm{p}<.01, \mathrm{MSe}=.0026$ ]. Of the 14 subjects, 12 had larger $A^{\prime}$ scores in the opposite-case condi- 
tion. The comparison between the combined control and opposite-case conditions and the same-case condition was also significant $[\mathrm{F}(1,13)=11.29$, $\mathrm{p}<.01$, MSe $=.0034]$. Since the opposite-case mean was significantly larger than the control mean, it is reasonable to conclude that the same-case mean was also significantly larger than the control mean. In fact, the $A^{\prime}$ scores for 13 of the 14 subjects were larger in the same-case condition than in the control condition. Thus, the same-case condition maintained a significant (in fact, an identical) advantage over the control condition with short and long onset asynchronies, while the opposite-case condition showed relative improvement (from no difference to a significant difference) with the longer onset asynchrony.

For the $\mathrm{B}^{\prime}{ }_{\mathrm{H}}$ means (Table 1, Row 4), there was a significant bias toward implicit-same responses in both the opposite- and same-case conditions $(p<.05$, $t$ tests) and no bias in the control condition. The comparison between the control condition and the combined opposite- and same-case conditions was significant $[F(1,13)=20.15, p<.001, \mathrm{MSe}=.0242]$, with the largest $B^{\prime}{ }_{H}$ score occurring in the control condition for 11 of the 14 subjects. However, the comparison between the opposite- and same-case conditions was not significant $[\mathrm{F}(1,13)=.21, \mathrm{p}>.05$, $\mathrm{MSe}=.0223 \mathrm{]}$, indicating that neither type of alternative induced a stronger bias.

\section{DISCUSSION}

Experiments 1 and 2 confirmed the predictions of the image-generation model regarding classification accuracy under marginal viewing conditions. A same-case prior alternative enhances classification accuracy with both short and long onset asynchronies, but an opposite-case alternative is effective only with the long onset asynchrony. The generality of the generation phenomenon has thus been extended by showing that it can be obtained in contexts other than reaction-time paradigms when similar onset asynchronies are examined. But does the image-generation model provide the best account of the classification data? An elaboration of the model will be presented here, and it will be compared to a likely alternative approach.

According to the image-generation model, the subject makes a same-different physical match of the target against the prior-alternative symbol whenever it is helpful. In the classification experiments, a physical match is helpful when the prior alternative is a letter, the representation of the alternative used in the match is the uppercase version of the letter, and the category of the target and the alternative is the same only if their identity is the same. In such cases, a physical match is advantageous because the name and category of the prior-alternative letter can be derived with perfect accuracy. Thus, target-letter classification accuracy depends solely on the accuracy of the physical-matching process. In general, physicalmatching accuracy should be greater than accuracy of target-letter identification using only the information from the target flash.

In the control condition, a physical match of the target and prior-alternative symbols is never helpful. Hence, the subject must identify the target letter on the basis of the information in the target flash and then use the name of the target letter to classify it. Direct classification without identification is unlikely, since the categories (first vs. second half of the alphabet) are relatively uncommon. (See Posner, 1973b, pp. 102-103, for a discussion of this point.) The identification process may involve feature matching of perceived fragments of the target letter against several visual patterns in memory, but the product is a target name which is then classified. Since correct identification of the target is uncertain, classification accuracy also suffers.

In the opposite-case condition, generation does not have time to occur with an onset asynchrony of 200 msec. Here too, then, the subject must identify the target letter from the information in the target flash, and accuracy is no better than in the control condition. However, with an onset asynchrony of $1,400 \mathrm{msec}$, generation does occur. Hence, the subject matches the target against an internal image of the uppercase version of the prior-alternative letter. Such matching is effective, resulting in better classification accuracy than in the control condition. Since accuracy in this condition is not as good as in the same-case condition, it must be assumed either that generation is not completed in $1,400 \mathrm{msec}$ or that the level of visual detail in the generated image is not as great as it is in the short-term visual memory of an actually presented uppercase prior-alternative letter.

In the same-case condition, the subject makes a physical match of the target against the prioralternative letter at both onset asynchronies. Since the subject cannot physically match the target and prior-alternative letters in the opposite-case condition with a 200 -msec onset asynchrony, the obvious alternative for the same-case condition is physical matching. Further, since the accuracy difference between the same-case and control conditions is identical for both onset asynchronies, there is little reason to infer a change in processing mode for the same-case condition with the 1,400 -msec onset asynchrony.

An alternative model that eliminates image generation assumes. that the subject attempts to match the names of the prior-alternative and target letters whenever they differ in case. Suppose that effective name matching is possible only when the subject is able to derive the name of the prior-alternative letter before the onset of the target letter. If $200 \mathrm{msec}$ is insufficient for deriving the name of the prior alternative, then there should be no difference between 
the opposite-case and control conditions with that onset asynchrony. With a 1,400 -msec onset asynchrony, the subject should have time to derive the name of the prior-alternative letter before the target flash. Thus, effective name matching should occur in the opposite-case condition, leading to an improvement in accuracy relative to the control condition. Physical matching in the same-case condition would account for its superior accuracy at both onset asynchronies.

This alternative model has a serious shortcoming. It fails to specify the mechanism whereby effective name matching leads to increased accuracy of targetletter classification. If identification of the target letter is equivalent to deriving its name, then name matching of target and prior alternative cannot occur until the target letter has been identified. However, once the name of the target letter is available, it can be classified directly without any need to match its name with that of the prior-alternative letter. Thus, the only way that having the name of the prior alternative can enhance accuracy of target classification is by enhancing target identification. If the name of the prior alternative enhances target identification by alerting the subject to look for critical features of the target, then a visual code is being primed, and that is a form of image generation. To eliminate image generation, the name of the prior alternative must enhance target identification accuracy without priming visual features or in any other way activating a visual code. It is difficult to imagine a credible model that meets this condition. Therefore, the image-generation model seems to provide the best explanation of the accuracy findings from these experiments.

It must be acknowledged that the size of the imagegeneration effect is quite small. The average gain in accuracy $\left(\mathrm{A}^{\prime}\right)$ in the opposite-case condition at $1,400 \mathrm{msec}$, after equating for performance in the control condition, is .03 . It is reasonable to question the importance of such a small effect. Three comments are in order. First, as noted above, the small gain may simply mean that generation is not completed in $1,400 \mathrm{msec}$. Second, performance gains attributable to generation in reaction-time studies are also small, typically less than $100 \mathrm{msec}$. This does not detract from their theoretical importance. Third, the present results agree with the earlier accuracy findings of Herzog (1976) and Pachella (1975). Methodological reservations about the earlier studies notwithstanding, all three studies point to the theoretically interesting conclusion that image generation can be demonstrated with an accuracy measure.

The present results also indicate that a prioralternative letter, regardless of its onset asynchrony or typecase, implicitly biases subjects toward reporting that the target letter has the same identity as the prior alternative. Herzog (1976) reported a similar finding. There has been a long-standing controversy in the selective-perception literature over whether set affects perception or simply biases responses. The present results suggest that stimulus set, in the form of a prior alternative, can affect both sensitivity (perceptual accuracy) and response bias.

\section{REFERENCES}

Egeth, H., \& Smrth, E. E. Perceptual selectivity in a visual recognition task. Journal of Experimental Psychology, 1967, 74, 543-549.

GRIER, J. B. Nonparametric indexes for sensitivity and bias: Computing formulas. Psychological Bulletin, 1971, 75, 424-429.

Gummerman, K. Selective perception and the number of alternatives. American Joumal of Psychology, 1971, 84, 173-179.

Herzog, T. R. Visual vs. verbal stimulus set in a letter-classification task. Memory \& Cognition, 1976, 4, 91-95.

KEPPEL, G. Design and analysis: $A$ researcher's handbook. Englewood Cliffs, N.J: Prentice-Hall, 1973.

Long, E. R., Reid, L. S., \& Henneman, R. H. An experimental analysis of set: Variables influencing the identification of ambiguous, visual stimulus objects. American Journal of Psychology, 1960, 73, 553-562.

Pachella, R. G. The effect of set on the tachistoscopic recognition of pictures. In P. Rabbit \& S. Dornic (Eds.), Attention and performance V. New York: Academic Press, 1975. Pp. 136-156. PosNer, M. I. Abstraction and the process of recognition. In G. H. Bower \& J. T. Spence (Eds.), The psychology of learning and motivation: Advances in research and theory (Vol. 3). New York: Academic Press, 1969. Pp. 43-100.

Posner, M. I. Coordination of internal codes. In W. G. Chase (Ed.), Visual information processing. New York: Academic Press, 1973. Pp. 35-73. (a)

Posner, M. I. Cognition: An introduction. Glenview, Ill: Scott. Foresman, 1973. (b)

Posner, M. I., Boies. S. J., Eichelman, W. H., \& Taylor, R. L. Retention of visual and name codes of single letters. Journal of Experimental Psychology Monograph, 1969, 79, 1-16.

Skanes, G.. \& Donderi, D. C. Stimulus set, response set, and word identification. Journal of Experimental Psychology, 1973, 99. 413-423.

TAYLOR, R. L., \& REILLY, S. Naming and other methods of decoding visual information. Joumal of Experimental Psychology, 1970, 83, 80-83.

(Received for publication April 5, 1976; revision accepted July 9 , 1976.) 